\title{
TRANSVERSAL MAPPINGS BETWEEN MANIFOLDS AND NON-TRIVIAL MEASURES ON VISIBLE PARTS
}

\author{
ESA JÄRVENPÄ̈̈ ${ }^{1}$, MAARIT JÄRVENPÄ̈̈ ${ }^{2}$, AND JUHO NIEMELÄ ${ }^{3}$
}

\begin{abstract}
Our main result explains in what sense typical visible parts of a set with large Hausdorff dimension are smaller than the set itself. This is achieved by generalizing the notation of sliced measures by means of transversal mappings, and by establishing a connection between dimensional properties of generalized slices and those of visible parts.
\end{abstract}

\section{BACKGROUND AND PRELIMINARY DISCUSSION}

Given integers $k$ and $d$ such that $0 \leq k \leq d-1$, and an affine $k$-plane $K$ in $\mathbb{R}^{d}$ (0-plane is simply a point), we use the notation $\operatorname{Proj}_{K}$ for the projection onto $K$. The following definition of visibility goes back to Urysohn [U] in the 1920's: Let $E \subset \mathbb{R}^{d}$ be compact. A point $a \in E$ is visible from $K$, if $a$ is the only point of $E$ in the line segment joining $a$ to $\operatorname{Proj}_{K}(a)$. The visible part of $E$ from $K$, denoted by $V_{K}(E)$, is the set of all points that are visible from $K$.

Visibility was investigated in connection with set theoretic problems by Nikodym in $[\mathrm{N}]$. The study of dimensional properties of visible parts, in turn, initiated in [JJMO]. Denoting by $\operatorname{dim}_{\mathrm{H}}$ the Hausdorff dimension, we have for almost all affine $k$-planes $K$ not intersecting $E$ that

$$
\operatorname{dim}_{\mathrm{H}}\left(V_{K}(E)\right)=\operatorname{dim}_{\mathrm{H}} E
$$

provided that $\operatorname{dim}_{\mathrm{H}} E \leq d-1$ [JJMO, Theorem 3.2]. On the other hand, under the assumption $\operatorname{dim}_{\mathrm{H}} E>d-1$, we have

$$
\operatorname{dim}_{\mathrm{H}}\left(V_{K}(E)\right) \geq d-1
$$

for almost all $k$-planes $K$ not intersecting $E$ [JJMO, Proposition 3.3]. It is not known whether the opposite inequality holds in (1.2). Some special examples of planar sets with Hausdorff dimension bigger than 1 are investigated in [JJMO]. In particular, it is shown that Hausdorff dimensions of all visible parts of a quasi-circle are equal to 1 . In [O] an upper bound bigger than 1 is verified for Hausdorff dimensions of

2000 Mathematics Subject Classification. 28A78, 28A80, 28A15.

Key words and phrases. Transversal mapping, generalized sliced measure, visible part, Hausdorff dimension.

MJ and JN acknowledge the support of the Academy of Finland, project \#48557. 
typical visible parts of connected and compact subsets of the plane. For further information on related topics, see [M3].

In this paper we approach the open question concerning the validity of the equality in (1.2) by proving the following weaker result:

Theorem 1.1. Let $E \subset \mathbb{R}^{d}$ be a compact set with $\operatorname{dim}_{\mathrm{H}} E>d-1$. Assume that $\mu$ is a Radon measure on $E$ such that $\mu(E)>0$ and $\operatorname{dim}_{\mathrm{H}} \mu>d-1$. Then $\mu\left(V_{K}(E)\right)=0$ for $\Gamma_{d, k}$-almost all $K \in A_{d, k}$ with $K \cap E=\emptyset$.

Here we use the notation $\operatorname{dim}_{H} \mu$ for the Hausdorff dimension of any finite measure $\mu$ on $\mathbb{R}^{d}$ (and later in section 2 on a metric space $X$ ), that is,

$$
\operatorname{dim}_{\mathrm{H}} \mu=\mu \text {-essinf } \underline{\operatorname{dim}}_{\mathrm{loc}} \mu(x)
$$

where

$$
\underline{\operatorname{dim}}_{\mathrm{loc}} \mu(x)=\liminf _{r \rightarrow 0} \frac{\log \mu(B(x, r))}{\log r} .
$$

(In (1.3) $B(x, r)$ is the closed ball with centre at $x$ and with radius $r>0$.) Moreover, the natural Radon measure on the space of affine $k$-planes of $\mathbb{R}^{d}$ is denoted by $\Gamma_{d, k}$ (see section 3 for definitions). Observe that, if (1.2) holds as an equality, Theorem 1.1 follows from it.

For the purpose of proving Theorem 1.1, we study a generalized version of the following notation of sliced measures introduced by Mattila in [M2]: Given an $k$-dimensional linear subspace $V$ of $\mathbb{R}^{d}$, let $V^{\perp}$ be the orthogonal complement of $V$. The slices of a finite Radon measure $\mu$ by affine planes $V_{a}=V+a$, where $a \in V^{\perp}$, are defined as weak limits of the normalized restriction measures

$$
\left.\mathcal{H}^{d-k}(B(a, \delta))^{-1} \mu\right|_{\operatorname{Proj}_{V^{\perp}}^{-1}(B(a, \delta))}
$$

as $\delta$ goes to 0 . (Here $\mathcal{H}^{d-k}$ is the $(d-k)$-dimensional Hausdorff measure and $\left.\mu\right|_{A}$ is the restriction of $\mu$ to a set $A$, in other words, $\left.\mu\right|_{A}(B)=\mu(A \cap B)$ for all $B \subset \mathbb{R}^{d}$.) The dimensional properties of these measures, which turn out to exist for $\mathcal{H}^{d-k}$-almost all $a \in V^{\perp}$, are investigated in $[\mathrm{JM}]$. In this paper we extend the notation of (1.4) by replacing the preimage of the projection by a preimage of a transversal mapping between manifolds. Studying dimensional properties of these generalized sliced measures in section 2 leads us to prove Theorem 1.1 in section 3. Our results in section 2 have a similar flavour to some of the results in [PS]: [PS, Theorem 7.7] may be regarded as a generalization of the projection results in [M1] for a parametrized family of transversal mappings, whilst section 2 extends the results of $[\mathrm{JM}]$ to a similar setting.

Our basic setting is as follows: Let $\left(L, \rho_{L}\right),\left(M, \rho_{M}\right)$, and $\left(N, \rho_{N}\right)$ be $l-, m$-, and $n$-dimensional smooth Riemannian manifolds, respectively. Assume that $l, m>n$ and $\Pi: L \times M \rightarrow N$ is a continuous function 
such that for $l=0,1,2$ there is a constant $c_{l}>1$ such that

$$
\left\|D_{\lambda}^{l} \Pi(\lambda, x)\right\| \leq c_{l}
$$

for all $\lambda \in L$ and $x \in M$. In (1.5) the derivative with respect to $\lambda$ is denoted by $D_{\lambda}$. Furthermore, suppose that there are finite collections $\{\phi, V\}$ and $\{\varphi, U\}$ of charts on $L$ and $N$, respectively, with the following property: there exists $R>0$ such that for all $\lambda \in L$ and $y \in N$

$$
B(\lambda, R) \subset V \text { and } B(y, R) \subset U
$$

for some $V$ and $U$. Assume also that the Lipschitz constants of the mappings $\varphi, \varphi^{-1}, \phi$, and $\phi^{-1}$ are uniformly bounded from above by a positive constant $K$.

We will restrict our consideration to the class of transversal mappings whose rôle becomes evident in the proof of Lemma 2.1.

Transversality. For $\lambda \in L$, let $\Pi_{\lambda}=\Pi(\lambda, \cdot)$. Suppose that the following form of transversality is satisfied: There is a constant $C_{t}>0$ such that for all $\lambda \in L$ and $x_{1} \neq x_{2} \in M$ for which $\rho_{N}\left(\Pi_{\lambda}\left(x_{1}\right), \Pi_{\lambda}\left(x_{2}\right)\right) \leq R$, the following condition holds: defining

$$
\Phi_{x_{1}, x_{2}}(\lambda)=\frac{\varphi \circ \Pi_{\lambda}\left(x_{1}\right)-\varphi \circ \Pi_{\lambda}\left(x_{2}\right)}{\rho_{M}\left(x_{1}, x_{2}\right)},
$$

the property

$$
\left|\Phi_{x_{1}, x_{2}}(\lambda)\right|<C_{t}
$$

implies that

$$
\operatorname{det}\left(D \Phi_{x_{1}, x_{2}}(\lambda) D \Phi_{x_{1}, x_{2}}(\lambda)^{T}\right)>C_{t}^{2} .
$$

Here $\varphi$ is as in (1.6), the derivative with respect to $\lambda$ is denoted by $D$, and $A^{T}$ is the transpose of a matrix $A$. Moreover, we assume that there exists a constant $\widetilde{L}>0$ such that

$$
\left\|\partial_{j} \partial_{k}\left(\Phi_{x_{1}, x_{2}}\right)_{i}(\lambda)\right\| \leq \widetilde{L}
$$

for all $j, k, i$, and for all $x_{1}, x_{2}$, and $\lambda$.

We continue by generalizing (1.4) in a way that is useful for our purposes.

Sliced measures determined by means of transversal mappings. Let $\mu$ be a Radon measure on $M$ and let $\lambda \in L$. Denote by $\mathcal{C}_{0}^{+}(M)$ the family of continuous non-negative functions on $M$ with compact support. As indicated below, it follows from the axiomatic theory of derivation in $[\mathrm{F}, 2.9]$ that for all $\psi \in \mathcal{C}_{0}^{+}(M)$ the limit

$$
\lim _{\delta \rightarrow 0} \frac{\left(\left(\Pi_{\lambda}\right)_{*} \nu_{\psi}\right)(B(y, \delta))}{\mathcal{H}^{n}(B(y, \delta))}=\lim _{\delta \rightarrow 0} \frac{1}{\mathcal{H}^{n}(B(y, \delta))} \int_{\Pi_{\lambda}^{-1}(B(y, \delta))} \psi d \mu
$$

exists and is finite for $\mathcal{H}^{n}$-almost all $y \in N$. Here $\nu_{\psi}(A)=\int_{A} \psi d \mu$ for all Borel sets $A \subset M$ and $f_{*} m$ is the image of a measure $m$ under a 
function $f: X \rightarrow Y$, that is, $f_{*} m(A)=m\left(f^{-1}(A)\right)$ for all $A \subset Y$. It is well-known that in a separable metric space $(X, \rho)$, satisfying a certain geometric condition described in $[F, 2.8 .9]$, the family

$$
\mathcal{V}=\{(x, B(x, r)) \mid x \in X, r>0\}
$$

is a $\mu$-Vitali relation for any locally finite Borel regular measure $\mu$ on $X[\mathrm{~F}$, Theorem 2.8.18] and [F, 2.8.16]. As indicated in [F, 2.8.9] this covers as a special case Riemannian $C^{k}$-manifolds $(k \geq 2)$ with the usual metrics. The existence of (1.10) follows now from [F, Theorem 2.9.5]. Using the separability of $\mathcal{C}_{0}^{+}(M)$ it may be shown that the exceptional set of points $y \in N$ in (1.10) is independent of the choice of $\psi$, and therefore we conclude from the Riesz representation theorem [M1, Theorem 1.16] that, given $\lambda \in L$, for $\mathcal{H}^{n}$-almost all $y \in N$ there is a Radon measure $\mu_{\lambda, y}$ such that

$$
\int \psi d \mu_{\lambda, y}=\lim _{\delta \rightarrow 0} \frac{1}{\mathcal{H}^{n}(B(y, \delta))} \int_{\Pi_{\lambda}^{-1}(B(y, \delta))} \psi d \mu
$$

for all $\psi \in \mathcal{C}_{0}^{+}(M)$. Clearly

$$
\text { spt } \mu_{\lambda, y} \subset \operatorname{spt} \mu \cap \Pi_{\lambda}^{-1}(\{y\}),
$$

where spt $\mu$ is the support of $\mu$.

Remark 1.2. (1) In (1.11) tranversality plays no rôle; only the continuity of $\Pi$ is needed.

(2) By [F, Lemma 2.9.6] the function $y \mapsto \int \psi d \mu_{\lambda, y}$ is $\mathcal{H}^{n}$-measurable for all $\psi \in \mathcal{C}_{0}^{+}(M)$.

The following disintegration formula holds for the measures $\mu_{\lambda, y}$ : Given a non-negative Borel function $f$ on $M$ with $\int f d \mu<\infty$, it follows from [F, Theorem 2.9.7] that for all Borel sets $B \subset N$

$$
\int_{B} \int f d \mu_{\lambda, y} d \mathcal{H}^{n} y \leq \int_{\Pi_{\lambda}^{-1}(B)} f d \mu
$$

Moreover, the equality holds in (1.13) provided that $\left(\Pi_{\lambda}\right)_{*} \mu$ is absolutely continuous with respect to $\mathcal{H}^{n}[\mathrm{~F}$, Theorem 2.9.2]. In this case we write $\left(\Pi_{\lambda}\right)_{*} \mu \ll \mathcal{H}^{n}$.

\section{Dimensional properties of Sliced measures Determined BY MEANS OF TRANSVERSAL MAPPINGS}

According to [JM, Theorem 3.8], if $\mu$ is a Radon measure on $\mathbb{R}^{d}$ with compact support and with $\operatorname{dim}_{\mathrm{H}} \mu>k$, then for almost all $(d-k)$ dimensional linear subspaces $V$ of $\mathbb{R}^{d}$ we have

$$
\operatorname{ess} \inf \left\{\operatorname{dim}_{\mathrm{H}} \mu_{V, a} \mid a \in V^{\perp} \text { with } \mu_{V, a}\left(\mathbb{R}^{d}\right)>0\right\}=\operatorname{dim}_{\mathrm{H}} \mu-k .
$$

In theorem 2.9 we prove an analogue of (2.1) for sliced measures determined by means of transversal mappings. Even though, for the purposes of this paper, Theorem 2.9 is being used in section 3 as a tool 
when investigating non-trivial measures on visible parts, we believe that it is also interesting on its own.

Our methods in this section combine those of [JM] and [PS]. We begin by proving the following generalization of [M1, Lemma 3.11]:

Lemma 2.1. Assume that $B \subset L$ is bounded. Then there are constants $c>0$ and $\delta_{0}>0$ such that for all $x_{1} \neq x_{2} \in M$ and $0<\delta<\delta_{0}$ we have

$$
\mathcal{H}^{l}\left(\left\{\lambda \in B \mid \rho_{N}\left(\Pi_{\lambda}\left(x_{1}\right), \Pi_{\lambda}\left(x_{2}\right)\right) \leq \delta\right\}\right) \leq c \delta^{n} \rho_{M}\left(x_{1}, x_{2}\right)^{-n} .
$$

Lemma 2.1 is obtained as an outcome of a sequence of lemmas (lemmas 2.2-2.7) in which the rôle of the transversality condition (1.8) is crucial. The basic idea of the proof is similar to that of [PS, Lemma 7.7]. Note that in the setting of [PS] both $L$ and $N$ are Euclidean spaces whereas $M$ is a metric space.

Let $R$ and $C_{t}$ be as in (1.6) and (1.7), respectively, and let $R_{1}=$ $R /\left(3 c_{1}\right)$ where $c_{1}$ is as in (1.5). Consider $\lambda_{0} \in L$ and $x_{1} \neq x_{2} \in M$ such that $\rho_{N}\left(\Pi_{\lambda_{0}}\left(x_{1}\right), \Pi_{\lambda_{0}}\left(x_{2}\right)\right) \leq R / 3$ and $\left|\Phi_{x_{1}, x_{2}}\left(\lambda_{0}\right)\right|<C_{t}$. Picking coordinates $\left(\eta_{1}, \ldots, \eta_{l}\right)$ in $B\left(\lambda_{0}, R_{1}\right)$ and applying transversality property (1.8) and Cauchy-Binet theorem, we find an $(n \times n)$-minor $A\left(\lambda_{0}\right)$ of $D \Phi_{x_{1}, x_{2}}\left(\lambda_{0}\right)$ with

$$
\left|\operatorname{det} A\left(\lambda_{0}\right)\right| \geq \tilde{c} C_{t} .
$$

Here $A\left(\lambda_{0}\right)$ is determined with respect to the coordinates $\left(\tilde{\eta}_{1}, \ldots, \tilde{\eta}_{n}\right)$ induced by $\left(\eta_{1}, \ldots, \eta_{n}, \ldots, \eta_{l}\right)$ and $\tilde{c}$ is a positive constant depending on $l$ and $n$. Given $\lambda=\left(\lambda_{1}, \ldots, \lambda_{n}, \lambda_{n+1}, \ldots, \lambda_{l}\right) \in B\left(\lambda_{0}, R_{1}\right)$, set

$$
H_{\lambda}=\left\{\lambda^{\prime} \in B\left(\lambda_{0}, R_{1}\right) \mid \lambda^{\prime}=\left(\lambda_{1}^{\prime}, \ldots, \lambda_{n}^{\prime}, \lambda_{n+1}, \ldots, \lambda_{l}\right)\right\} .
$$

Defining a function $\psi_{\lambda}: H_{\lambda} \rightarrow \mathbb{R}^{n}$ by

$$
\psi_{\lambda}\left(\lambda^{\prime}\right)=\Phi_{x_{1}, x_{2}}\left(\lambda^{\prime}\right)
$$

for all $\lambda^{\prime} \in H_{\lambda}$, the following lemma holds (observe that by (1.5) and the choice of $R_{1}$ the function $\psi_{\lambda}$ is well defined):

Lemma 2.2. There exists $0<R_{0} \leq R_{1}$ which is independent of $\lambda_{0}$ such that the following properties hold:

(1) For all $\lambda \in B\left(\lambda_{0}, R_{0}\right)$ the absolute values of the singular values of $D_{\lambda} \psi_{\lambda}$ are bounded below and above by positive constants that do not depend on $\lambda_{0}$ and $\lambda$.

(2) For all $\lambda \in B\left(\lambda_{0}, R_{0}\right)$ the function $\psi_{\lambda}: B(\lambda, \widetilde{R} / 3) \cap H_{\lambda} \rightarrow$ $\psi_{\lambda}\left(B(\lambda, \widetilde{R} / 3) \cap H_{\lambda}\right)$ is a diffeomorphism, and

$$
\psi_{\lambda}\left(B(\lambda, \rho) \cap H_{\lambda}\right) \supset B\left(\psi_{\lambda}(\lambda), d \rho\right)
$$

for all $0<\rho \leq \widetilde{R}$. Here $\widetilde{R}$ and $d$ are independent of $\lambda_{0}$ and $\lambda$.

Proof. (1) From (1.9) we see that the absolute values of the singular values of $\left(D \psi_{\lambda_{0}}\right)\left(\lambda_{0}\right)$ are bounded above by a constant $C(\widetilde{L})$. Furthermore, since $\left(D \psi_{\lambda_{0}}\right)\left(\lambda_{0}\right)=A\left(\lambda_{0}\right)$, inequality (2.2) implies that the 
absolute values of the singular values of $\left(D \psi_{\lambda_{0}}\right)\left(\lambda_{0}\right)$ are bounded below by the constant $\tilde{c} C_{t} / C(\widetilde{L})^{n-1}$. The claim follows since the function $\lambda \mapsto\left(D \Phi_{x_{1}, x_{2}}\right)(\lambda)$ is uniformly continuous by $(1.9)$.

(2) Defining a function $\widetilde{\psi}_{\lambda}: H_{\lambda} \rightarrow \mathbb{R}^{n}$ by

$$
\widetilde{\psi}_{\lambda}\left(\lambda^{\prime}\right)=\left(D \psi_{\lambda}\right)(\lambda)^{-1} \psi_{\lambda}\left(\lambda^{\prime}\right)
$$

and using the uniform continuity of the function $\lambda \mapsto\left(D \Phi_{x_{1}, x_{2}}\right)(\lambda)$, we see that the derivative $D \widetilde{\psi}_{\lambda}\left(\lambda^{\prime}\right)=D \psi_{\lambda}(\lambda)^{-1} D \psi_{\lambda}\left(\lambda^{\prime}\right)$ is close to the identity in some ball $B(\lambda, \widetilde{R}) \cap H_{\lambda}$. (Here $\widetilde{R}$ does not depend on $\lambda_{0}$ and $\lambda$.) Applying [PS, Lemma 7.6] gives that $\widetilde{\psi}_{\lambda}: B(\lambda, \widetilde{R} / 3) \cap$ $H_{\lambda} \rightarrow \widetilde{\psi}_{\lambda}\left(B(\lambda, \widetilde{R} / 3) \cap H_{\lambda}\right)$ is a diffeomorphism, and $\widetilde{\psi}_{\lambda}\left(B(\lambda, \rho) \cap H_{\lambda}\right) \supset$ $B\left(\widetilde{\psi}_{\lambda}(\lambda), \rho / 2\right)$ for all $0<\rho \leq \widetilde{R}$. This in turn completes the proof of (2). Note that the constant $d$ depends on the lower bound of the absolute values of the eigenvalues of $D \psi_{\lambda}(\lambda)$, and therefore, by (1), it is independent of $\lambda_{0}$ and $\lambda$.

According to the next lemma, there is a zero of the function $\Phi_{x_{1}, x_{2}}$ close to each parameter $\lambda$ for which $\left|\Phi_{x_{1}, x_{2}}(\lambda)\right|$ is small enough.

Lemma 2.3. Suppose that $x_{1} \neq x_{2} \in M$ and $\lambda \in L$ such that $\rho_{N}\left(\Pi_{\lambda}\left(x_{1}\right), \Pi_{\lambda}\left(x_{2}\right)\right) \leq R / 3$. Then, given any $0<\delta<\min \left\{C_{t}, \widetilde{R} d / 4\right\}$, the condition $\left|\Phi_{x_{1}, x_{2}}(\lambda)\right|<\delta$ implies the existence of $\tilde{\lambda} \in L$ such that $\Phi_{x_{1}, x_{2}}(\tilde{\lambda})=0$ and $\rho_{L}(\lambda, \tilde{\lambda}) \leq \delta / d \leq \widetilde{R} / 4$.

Proof. Lemma 2.2 gives the inclusion

$$
\psi_{\lambda}\left(B(\lambda, \delta / d) \cap H_{\lambda}\right) \supset B\left(\Phi_{x_{1}, x_{2}}(\lambda), \delta\right)
$$

which implies the claim.

Given any $x_{1} \neq x_{2} \in M$ and $\tilde{\lambda} \in L$ with $\Phi_{x_{1}, x_{2}}(\tilde{\lambda})=0$, define an $(l-n)$-dimensional submanifold $L_{x_{1}, x_{2}}(\tilde{\lambda})$ of $L$ as follows:

$$
L_{x_{1}, x_{2}}(\tilde{\lambda})=\Phi_{x_{1}, x_{2}}^{-1}(0) \cap B(\tilde{\lambda}, \widetilde{R}) .
$$

Lemma 2.4. Let $x_{1} \underset{\sim}{\neq} x_{2} \in M$ and $\tilde{\lambda} \in L$ such that $\Phi_{x_{1}, x_{2}}(\tilde{\lambda})=0$. Then for all $0<\delta<\widetilde{R} d / 4$ we have

$$
\left\{\lambda \in B(\tilde{\lambda}, \widetilde{R} / 2)|| \Phi_{x_{1}, x_{2}}(\lambda) \mid \leq \delta\right\} \subset \bigcup_{\lambda^{\prime} \in L_{x_{1}, x_{2}}(\tilde{\lambda})} B\left(\lambda^{\prime}, \delta / d\right) .
$$

Proof. We may assume that $\widetilde{R} \leq 2 R_{0}$. Then $\Phi_{x_{1}, x_{2}}(\lambda)$ is defined for all $\lambda \in B(\tilde{\lambda}, \widetilde{R} / 2)$. Letting $\lambda \in B(\tilde{\lambda}, \widetilde{R} / 2)$ such that $\left|\Phi_{x_{1}, x_{2}}(\lambda)\right|<\delta$ and using Lemma 2.3, we find $\lambda^{\prime} \in B(\lambda, \delta / d) \cap H_{\lambda}$ such that $\Phi_{x_{1}, x_{2}}\left(\lambda^{\prime}\right)=0$. Since $\lambda^{\prime} \in B(\tilde{\lambda}, \widetilde{R})$, the claim follows.

Let $\delta_{0}=\min \left\{C_{t}, \widetilde{R} d / 4, R / 3\right\}$. Consider $x_{1} \neq x_{2} \in M$. For the proof of Lemma 2.1 we may assume that $B \subset V$ for some $V$ defined in (1.6) and $K \delta \leq \delta_{0} \rho_{M}\left(x_{1}, x_{2}\right)$. Recalling that the Lipschitz constants of 
$\varphi, \varphi^{-1}, \phi$, and $\phi^{-1}$ are uniformly bounded above by $K$, and applying Lemma 2.3, one finds a constant $N$ (depending only on $K, \widetilde{R}, l$, and the diameter of $B)$ and $\tilde{\lambda}_{1}, \ldots, \tilde{\lambda}_{N} \in L$ with $\Phi_{x_{1}, x_{2}}\left(\tilde{\lambda}_{i}\right)=0$ such that

$$
\begin{aligned}
\{\lambda \in B \mid & \left.\rho_{N}\left(\Pi_{\lambda}\left(x_{1}\right), \Pi_{\lambda}\left(x_{2}\right)\right) \leq \delta\right\} \\
& \subset \bigcup_{i=1}^{N}\left\{\lambda \in B\left(\tilde{\lambda}_{i}, \widetilde{R} / 2\right)|| \Phi_{x_{1}, x_{2}}(\lambda) \mid \leq K \delta / \rho_{M}\left(x_{1}, x_{2}\right)\right\} .
\end{aligned}
$$

Defining for all $\tilde{\lambda} \in L$ with $\Phi_{x_{1}, x_{2}}(\tilde{\lambda})=0$ and $\delta>0$

$$
N\left(L_{x_{1}, x_{2}}(\tilde{\lambda}), \delta / d\right)=\bigcup_{\lambda^{\prime} \in L_{x_{1}, x_{2}}(\tilde{\lambda})} B\left(\lambda^{\prime}, \delta / d\right),
$$

we see from Lemma 2.4 that Lemma 2.1 is an immediate consequence of the following result.

Lemma 2.5. Given $x_{1} \neq x_{2} \in M$ and $\tilde{\lambda} \in L$ with $\Phi_{x_{1}, x_{2}}(\tilde{\lambda})=0$ and $0<\delta<\delta_{0}$, we have

$$
\mathcal{H}^{l}\left(N\left(L_{x_{1}, x_{2}}(\tilde{\lambda}), \delta / d\right)\right) \leq c \delta^{n}
$$

Here the constant $c$ is independent of $\tilde{\lambda}$ and $\delta$.

Lemmas 2.6 and 2.7, in turn, lead to Lemma 2.5.

Lemma 2.6. Let $x_{1} \neq x_{2} \in M$ and $\tilde{\lambda} \in L$ such that $\Phi_{x_{1}, x_{2}}(\tilde{\lambda})=0$. Then there exists $\alpha>0$ which is independent of $\tilde{\lambda}$ such that for all $\lambda \in B(\tilde{\lambda}, \widetilde{R}), h \in T_{\lambda} H_{\lambda}\left(T_{\lambda} H_{\lambda}\right.$ is the tangent space of $H_{\lambda}$ at $\left.\lambda\right)$, and $y \in T_{\lambda} L_{x_{1}, x_{2}}(\tilde{\lambda})$ with $\|h\|=\|y\|=1$, we have $\varangle(h, y) \geq \alpha$. (Here $\varangle(h, y)$ is the angle between $h$ and $y$.)

Proof. Let $p=h-y \in T_{\lambda} L$. Denoting by $c$ the lower bound given in Lemma 2.2 (a) and using (1.9), we get

$$
c \leq\left|D_{\lambda} \psi_{\lambda}(\lambda) h\right|=\left|\left(D_{\lambda} \Phi_{x_{1}, x_{2}}\right)(\lambda) h\right|=\left|\left(D_{\lambda} \Phi_{x_{1}, x_{2}}\right)(\lambda) p\right| \leq c(\widetilde{L})\|p\|
$$

since $\left(D_{\lambda} \Phi_{x_{1}, x_{2}}\right)(\lambda) y=0$. This completes the proof.

For $x_{1} \neq x_{2} \in M$ and $\tilde{\lambda}=\left(\tilde{\lambda}_{1}, \ldots, \tilde{\lambda}_{l}\right) \in L$ with $\Phi_{x_{1}, x_{2}}(\tilde{\lambda})=0$, define

$$
V_{\tilde{\lambda}}=\left\{\lambda^{\prime} \in L \mid \lambda^{\prime}=\left(\tilde{\lambda}_{1}, \ldots, \tilde{\lambda}_{n}, \lambda_{n+1}^{\prime}, \ldots, \lambda_{l}^{\prime}\right)\right\} .
$$

From Lemma 2.6 we immediately get the following result which, in turn, completes the proof of Lemma 2.1 by verifying Lemma 2.5.

Lemma 2.7. Given $x_{1} \neq x_{2} \in M, \tilde{\lambda} \in L$ with $\Phi_{x_{1}, x_{2}}(\tilde{\lambda})=0$ and $0<\delta<\delta_{0}$, there is an integer $I \leq c(l, n)(\widetilde{R} / \delta)^{l-n}$ and a covering of $V_{\tilde{\lambda}} \cap B(\tilde{\lambda}, \widetilde{R})$ with balls $B_{1}, \ldots, B_{I}$ of radius $\delta$ which induces a covering of $N\left(L_{x_{1}, x_{2}}(\tilde{\lambda}), \delta / d\right)$ with balls $\widetilde{B}_{1}, \ldots, \widetilde{B}_{I}$ of radius $c(\alpha) \delta$.

The rôle of Lemma 2.1 is crucial in the proof of the following result which combines the methods from [FM, Lemma 4.1] and [M1, Theorem 9.7]. For reader's convenience we will give a brief outline of the proof in our setting. 
Given locally finite measures $\nu_{1}$ and $\nu_{2}$ on a metric space $(X, \rho)$, denote by $\underline{\mathrm{D}}\left(\nu_{1}, \nu_{2}, x\right)$ the lower derivative of $\nu_{1}$ with respect to $\nu_{2}$ at a point $x \in X$, that is,

$$
\underline{\mathrm{D}}\left(\nu_{1}, \nu_{2}, x\right)=\liminf _{r \rightarrow 0} \frac{\nu_{1}(B(x, r))}{\nu_{2}(B(x, r))} .
$$

Moreover, for $s \geq 0$, the $s$-energy $I_{s}(\nu)$ of a measure $\nu$ is defined as

$$
I_{s}(\nu)=\iint \rho(x, y)^{-s} d \nu(x) d \nu(y) .
$$

Lemma 2.8. Let $\mu$ be a Radon measure on $M$ with compact support. Then the following properties hold:

(1) We have $\left(\Pi_{\lambda}\right)_{*} \mu \ll \mathcal{H}^{n}$ if and only if $\underline{\mathrm{D}}\left(\left(\Pi_{\lambda}\right)_{*} \mu, \mathcal{H}^{n}, y\right)<\infty$ for $\left(\Pi_{\lambda}\right)_{*} \mu$-almost all $y \in N$.

(2) Both $\operatorname{dim}_{\mathrm{H}} \mu>n$ and $I_{n}(\mu)<\infty$ imply that $\left(\Pi_{\lambda}\right)_{*} \mu \ll \mathcal{H}^{n}$ for $\mathcal{H}^{l}$-almost all $\lambda \in L$.

Proof. (1) Supposing that $\left(\Pi_{\lambda}\right)_{*} \mu \ll \mathcal{H}^{n}$, the finiteness of the lower derivative follows directly from (1.11). On the other hand, assume that $\underline{\mathrm{D}}\left(\left(\Pi_{\lambda}\right)_{*} \mu, \mathcal{H}^{n}, y\right)<\infty$ for $\left(\Pi_{\lambda}\right)_{*} \mu$-almost all $y \in N$. Given $A \subset N$ with $\mathcal{H}^{n}(A)=0$, it is sufficient to prove that

$$
\left(\Pi_{\lambda}\right)_{*} \mu\left\{y \in A \mid \underline{\mathrm{D}}\left(\left(\Pi_{\lambda}\right)_{*} \mu, \mathcal{H}^{n}, y\right)<c\right\}=0
$$

for all $0<c<\infty$. This follows from [F, Lemma 2.9.3] by choosing $\alpha=$ $\phi=\left(\Pi_{\lambda}\right)_{*} \mu$ and $\beta=\mathcal{H}^{n}$ therein. (Recall that the family $\{(y, B(y, r)) \mid$ $y \in N, r>0\}$ is both a $\left(\Pi_{\lambda}\right)_{*} \mu$-Vitali relation and a $\mathcal{H}^{n}$-Vitali relation, and therefore this choice is possible.)

(2) Let $\operatorname{dim}_{\mathrm{H}} \mu>s>n$. Defining for all $i=1,2, \ldots$ a restriction measure $\mu_{i}=\left.\mu\right|_{M_{i}}$ where

$$
M_{i}=\left\{x \in M \mid \mu(B(x, r)) \leq i r^{s} \text { for all } r>0\right\},
$$

we have $I_{n}\left(\mu_{i}\right)<\infty$. Moreover, it follows from Fatou's lemma, Fubini's theorem, and Lemma 2.1 that

$$
\int_{B} \int \underline{\mathrm{D}}\left(\left(\Pi_{\lambda}\right)_{*} \mu_{i}, \mathcal{H}^{n}, y\right) d\left(\Pi_{\lambda}\right)_{*} \mu_{i}(y) d \mathcal{H}^{l}(\lambda) \leq c I_{n}\left(\mu_{i}\right)
$$

for all bounded sets $B \subset L$. From (1) we get $\left(\Pi_{\lambda}\right)_{*} \mu_{i} \ll \mathcal{H}^{n}$ for $\mathcal{H}^{l}$ almost all $\lambda \in L$. This settles the claim since $x \in \cup_{i} M_{i}$ for $\mu$-almost all $x \in M$.

Now we are ready to state the main result of this section.

Theorem 2.9. Let $\mu$ be a Radon measure on $M$ with compact support. Assuming that $\operatorname{dim}_{\mathrm{H}} \mu>n$, we have

$\mathcal{H}^{n}$-ess inf $\left\{\operatorname{dim}_{\mathrm{H}} \mu_{\lambda, y} \mid y \in N\right.$ with $\left.\mu_{\lambda, y}(M)>0\right\}=\operatorname{dim}_{\mathrm{H}} \mu-n$

for $\mathcal{H}^{l}$-almost all $\lambda \in L$. 
Proof. Having proved Lemmas 2.1 and 2.8, the methods from the proof of [JM, Theorem 3.8] can be extended in a straightforward manner to our setting.

\section{Non-trivial MEASURES ON Visible PARTS}

In this section we prove Theorem 1.1. We begin by introducing the notation needed for this purpose.

Let $k$ and $d$ be integers such that $0 \leq k \leq d-1$. The Grassmann manifold of linear $k$-dimensional subspaces of $\mathbb{R}^{d}$ and the space of affine $k$-dimensional subspaces of $\mathbb{R}^{d}$ are denoted by $G_{d, k}$ and $A_{d, k}$, respectively. (A 0 -plane is simply a point.) Letting $\gamma_{d, k}$ be the unique orthogonally invariant Radon probability measure on $G_{d, k}$, and defining for all Borel sets $A \subset A_{d, k}$

$$
\Gamma_{d, k}(A)=\int \mathcal{H}^{d-k}\left(\left\{a \in V^{\perp} \mid V+a \in A\right\}\right) d \gamma_{d, k}(V),
$$

gives a Radon measure $\Gamma_{d, k}$ on $A_{d, k}$. Observe that both $G_{d, k}$ and $A_{d, k}$ are smooth Riemannian manifolds, and $\Gamma_{d, k}$ is equivalent to $\mathcal{H}^{s}$ with $s=\operatorname{dim} A_{d, k}$.

Visible parts. The visible part of a compact set $E \subset \mathbb{R}^{d}$ from an affine subspace $K \in A_{d, k}$ with $E \cap K=\emptyset$ is

$$
V_{K}(E)=\left\{x \in E \mid\left[\operatorname{Proj}_{K}(x), x\right] \cap E=\{x\}\right\} .
$$

Here $\operatorname{Proj}_{K}(x)=\operatorname{Proj}_{V}(x)+a$ is the closest point to $x$ on the affine plane $K=V+a$, where $V \in G_{d, k}$ and $a \in V^{\perp}$, and $[x, y]$ is the line segment between $x$ and $y$.

The following remark is needed when proving Theorem 1.1 as a consequence of Theorem 2.9:

Remark 3.1. (a) The visible part $V_{K}(E)$ is a Borel set as the graph of a lower semi-continuous function [JJMO, Remark 2.2 (a)].

(b) Let $B \subset M$ be a Borel set and $\lambda \in L$ such that $\left(\Pi_{\lambda}\right)_{*} \mu \ll \mathcal{H}^{n}$. Then, under the assumptions of section 1 , we have

$$
\left.\mu_{\lambda, y}\right|_{B}=\left(\left.\mu\right|_{B}\right)_{\lambda, y}
$$

for $\mathcal{H}^{n}$-almost all $y \in N$. This can be verified using similar arguments as in [JM, Lemma 3.2].

Proof of Theorem 1.1. Consider a compact set $E \subset \mathbb{R}^{d}$ with $\operatorname{dim}_{\mathrm{H}} E>$ $d-1$. Let $\mu$ be a Radon measure on $E$ such that $\mu(E)>0$ and $\operatorname{dim}_{\mathrm{H}} \mu>d-1$. Given $\varepsilon>0$, let $A_{d, k}(E, \varepsilon)$ consist of those $K \in A_{d, k}$ for which $\operatorname{dist}(K, E)>\varepsilon$. Defining for all $K \in A_{d, k}(E, \varepsilon)$

$$
N_{K}=\left\{x \in \mathbb{R}^{d} \mid \operatorname{dist}(x, K)=\varepsilon\right\},
$$

the assumptions of section 1 are satisfied for the natural projection $\Pi_{K}: \mathbb{R}^{d} \rightarrow N_{K}$; the transversality of $\Pi_{K}$ follows from [JJMO, (3.4)]. Since $\operatorname{dim}_{\mathrm{H}} \mu>d-1$, Lemma 2.8 (2) gives that $\left(\Pi_{K}\right)_{*} \mu \ll \mathcal{H}^{d-1}$ for 
$\Gamma_{d, k}$-almost all $K \in A_{d, k}(E, \varepsilon)$. Defining $D$ as the set of such affine $k$-planes and using Remark 3.1 (b), we get for all $K \in D$ and for all Borel sets $B \subset E$

$$
\left.\mu_{K, y}\right|_{B}=\left(\left.\mu\right|_{B}\right)_{K, y}
$$

for $\mathcal{H}^{d-1}$-almost all $y \in N_{K}$. Combining this with Lemma 2.9 implies the existence of $\widetilde{D} \subset D$ with $\Gamma_{d, k}\left(A_{d, k}(E, \varepsilon) \backslash \widetilde{D}\right)=0$ such that for all Borel sets $B \subset E$ we have

$$
\begin{aligned}
& \mathcal{H}^{d-1} \text {-essinf }\left\{\operatorname{dim}_{\mathrm{H}}\left(\left.\mu\right|_{B}\right)_{K, y} \mid y \in N_{K} \text { with }\left(\left.\mu\right|_{B}\right)_{K, y}\left(\mathbb{R}^{d}\right)>0\right\} \\
& \geq \mathcal{H}^{d-1} \text {-ess } \inf \left\{\operatorname{dim}_{\mathrm{H}} \mu_{K, y} \mid y \in N_{K} \text { with } \mu_{K, y}\left(\mathbb{R}^{d}\right)>0\right\} \\
& =\operatorname{dim}_{\mathrm{H}} \mu-(d-1)>0
\end{aligned}
$$

for all $K \in \widetilde{D}$.

The final step is to conclude that if $K \in A_{d, k}(E, \varepsilon)$ such that

$$
\mu\left(V_{K}(E)\right)>0,
$$

then $K \notin \widetilde{D}$. Assume to the contrary that $K \in \widetilde{D}$. Recalling remark 3.1 (a) and applying (3.1) with $B=V_{K}(E)$, gives

$$
\operatorname{dim}_{\mathrm{H}}\left(V_{K}(E) \cap \Pi_{K}^{-1}(\{y\})\right)>0
$$

for $\mathcal{H}^{d-1}$-almost all $y \in N_{K}$ with $\left(\left.\mu\right|_{V_{K}(E)}\right)_{K, y}\left(\mathbb{R}^{d}\right)>0$. Noting that the set $V_{K}(E) \cap \Pi_{K}^{-1}(\{y\})$ contains at most one point for all $y \in N_{K}$, this gives a contradiction, since

$$
\mathcal{H}^{d-1}\left(\left\{y \in N_{K} \mid\left(\left.\mu\right|_{V_{K}(E)}\right)_{K, y}\left(\mathbb{R}^{d}\right)>0\right\}\right)>0
$$

by (1.13). The claim follows letting $\varepsilon$ tend to 0 along a sequence.

\section{REFERENCES}

[FM] K. J. Falconer and P. Mattila, Packing dimensions of projections, Math. Proc. Cambridge Phil. Soc. 119 (1996), 695-713.

[F] H. Federer, Geometric measure theory, Springer Verlag, Heidelberg, 1996.

[JJMO] E. Järvenpää, M. Järvenpää, P. MacManus, and T. C. O'Neil, Visible parts and dimensions, Nonlinearity 16 (2003), 803-818.

[JM] M. Järvenpää and P. Mattila, Hausdorff and packing dimensions and sections of measures, Mathematika (1998), 55-77.

[M1] P. Mattila, Geometry of sets and measures in Euclidean spaces: fractals and rectifiability, Cambridge University Press, Cambridge, 1995.

[M2] P. Mattila, Hausdorff dimension, orthogonal projections and intersections with planes, Ann. Acad. Sci. Fenn. Ser. A I Math 1 (1975), 227-244.

[M3] P. Mattila, Hausdorff dimension, projections, and Fourier transform, to appear in Publ. Mat.

[N] O. Nikodym, Sur la points linéairement accessibles des ensembles plans, Fundamenta Mathematicae 10 (1927), 116-168.

[O] T. C. O'Neil, The Hausdorff dimension of the visible sets of connected compact sets, a preprint.

[PS] Y. Peres and W.Schlag, Smoothness of projections, Bernoulli convolutions, and the dimension of exceptions, Duke Math. J. (2000), 193-251.

[U] P. Urysohn, Problème 29, Fundamenta Mathematicae 5 (1923), 337. 
Department of Mathematics and Statistics, P.O. Box 35, FIN-40014 UNIVERSiTy OF JYVÄSKYlä, FINLAND ${ }^{1,2,3}$

E-mail address: esaj@maths.jyu.fi ${ }^{1}$

E-mail address: amj@maths.jyu.fi ${ }^{2}$

E-mail address: juniemel@maths.jyu.fi ${ }^{3}$ 